\section{REVIEW ARTICLE}

S.K. Thawait

V. Chaudhry

G.K. Thawait

K.C. Wang

A. Belzberg

J.A. Carrino

A. Chhabra

\title{
High-Resolution MR Neurography of Diffuse Peripheral Nerve Lesions
}

SUMMARY: High-resolution MR imaging of peripheral nerves is becoming more common and practical with the increasing availability of $3 T$ magnets. There are multiple reports of MR imaging of peripheral nerves in compression and entrapment neuropathies. However, there is a relative paucity of literature on MRN appearance of diffuse peripheral nerve lesions. We attempted to highlight the salient imaging features of myriad diffuse peripheral nerve disorders and imaging techniques for MRN. Using clinical and pathologically proved relevant examples, we present the MRN appearance of various types of diffuse peripheral nerve lesions, such as traumatic, inflammatory, infectious, hereditary, radiationinduced, neoplastic, and tumor variants.

ABBREVIATIONS: CIDP $=$ chronic inflammatory demyelinating polyneuropathy; $\mathrm{CMT}=$ CharcotMarie-Tooth; fat sat $=$ fat saturated; FLAIR $=$ fluid-attenuated inversion recovery; FLH $=$ fibrolipomatous hamartoma; GBS = Guillain Barré syndrome; CMT/HSMN = Charcot-Marie-Tooth/hereditary motor and sensory neuropathy; MMN = multifocal motor neuropathy; MPNST = malignant peripheral nerve sheath tumor; $\mathrm{MRN}=\mathrm{MR}$ neurography; NF1 = neurofibromatosis type 1 ; $\mathrm{NL}=$ neurolymphomatosis; SE = spin-echo; SNR = signal-to-noise ratio; SPACE = sampling perfection with application-optimized contrasts by using different flip angle evolutions; SPAIR = spectralattenuated inversion recovery; STIR = short-tau inversion recovery; T1WI = T1-weighted imaging; T1WIFS = T1-weighted fat-saturated imaging; T2WI = T2-weighted imaging; T2WIFS = T2weighted fat-saturated imaging

$\mathbf{H}^{\mathrm{i}}$ gh-resolution MR imaging of peripheral nerves is becoming more common and practical with increasing availability of $3 \mathrm{~T}$ magnets. These magnets provide high SNR, which can be used for a quicker acquisition time as well as higher image contrast and resolution. There have been multiple reports of MR imaging of peripheral nerves in compression and entrapment neuropathies. ${ }^{1-3}$ However, there is a relative paucity of literature on the MRN appearance of diffuse peripheral nerve lesions. ${ }^{4}$ These lesions seen on MR imaging present a diagnostic dilemma because a long list of pathologies could be causing them. We attempt to highlight the salient imaging features of myriad diffuse peripheral nerve disorders and to describe a diagnostic approach to these lesions on the basis of the available literature and our experience in this area. Various clinical and pathologically proved relevant examples of these pathologies are illustrated.
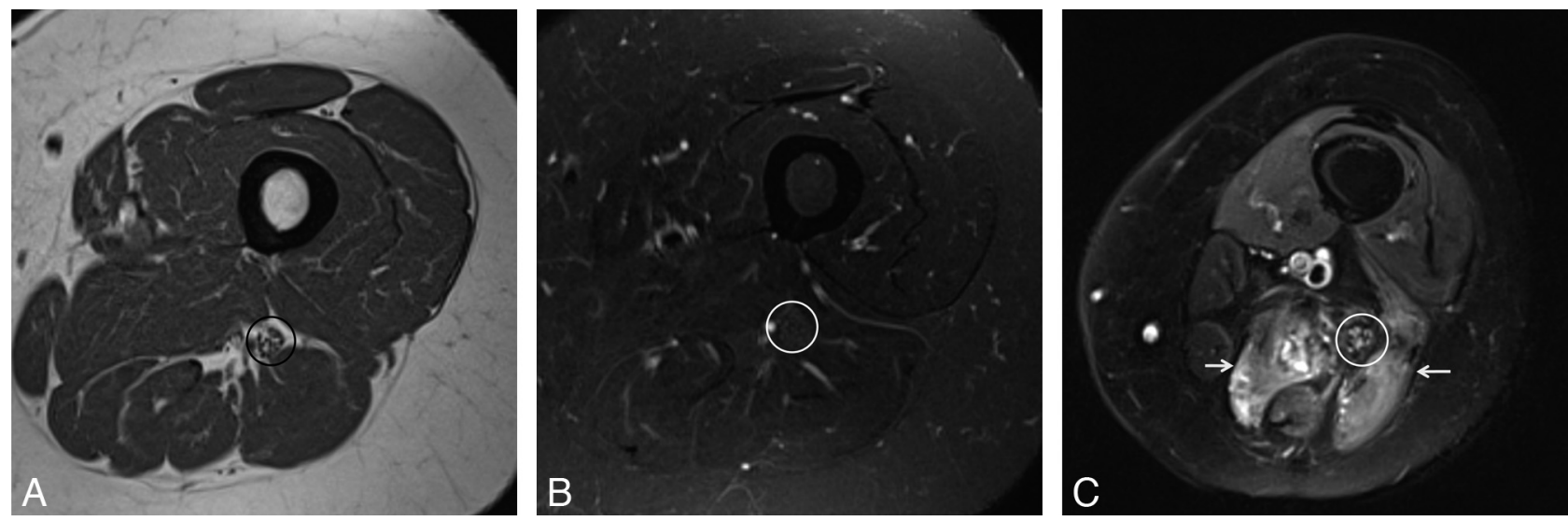

Fig 1. Normal $(A$ and $B)$ versus abnormal $(C)$ MRN appearance of the sciatic nerve. Axial T1WI $(A)$ and T2WI $(B)$ sections at the level of the midthigh show low-intermediate nerve signal intensity (circles). T1WI demonstrates fat planes delineating the normal nerve (perineural fat). C, Axial STIR SPACE at the level of the thighs shows an abnormal sciatic nerve. Notice the enlarged size and T2 hyperintensity of the fascicles. The dark rim of perineural fat is also disrupted. Also note posterior compartment denervation muscle edema (arrows).

From the Russell H. Morgan Department of Radiology and Radiological Science (S.K.T. G.K.T., K.C.W., J.A.C., A.C.), Department of Neurology (V.C.), and Department of Neurosurgery (A.B.), Johns Hopkins Hospital, Baltimore, Maryland.

K.C.W. was supported by the Radiological Society of North America Research and Education Foundation Fellowship Training Grant FT0904 and the Walter and Mary Ciceric Research Award.

Paper previously presented as an Electronic Scientific Exhibit at: Annual Meeting of the American Society of Neuroradiology, May 15-20, 2010; Boston, Massachusetts.
Please address correspondence to Avneesh Chhabra, MD, Russell H. Morgan Department of Radiology and Radiological Science, Johns Hopkins Hospital, 601 North Caroline St, JHOC 3262, Baltimore, MD 21287; e-mail: achhabr6@jhmi.edu

Indicates open access to non-subscribers at www.ajnr.org

http://dx.doi.org/10.3174/ajnr.A2257 

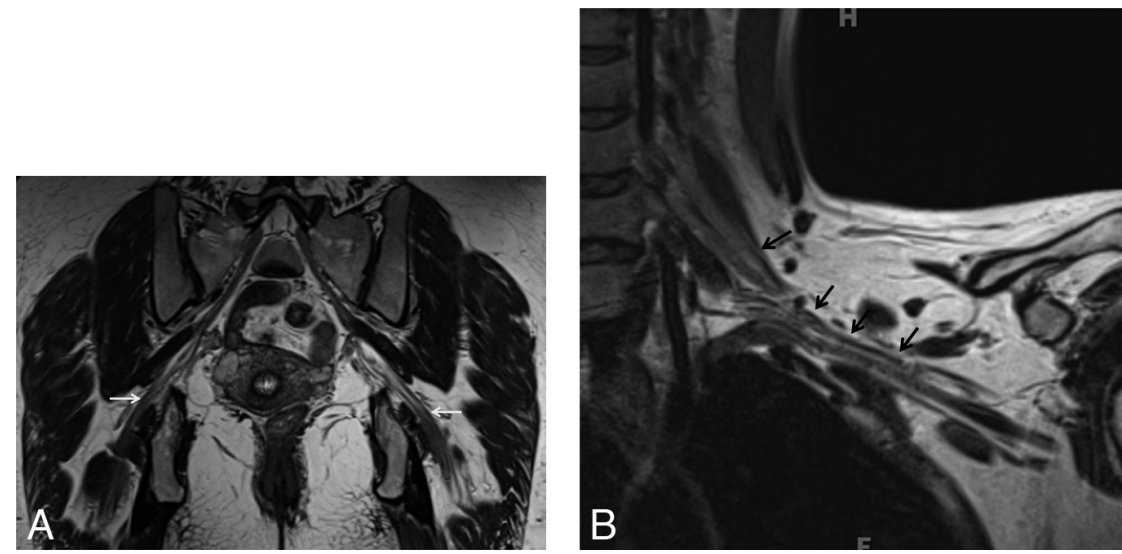

Fig 2. Normal peripheral nerves as seen on SPACE sequences. Coronal 3D T2 SPACE image of the pelvis $(A)$ and coronal 3D T2 SPACE image of the left shoulder (B) depict the normal smooth course of the sciatic nerve and brachial plexus respectively (arrows). Note the smooth course outlined clearly by perineural fat planes.

\begin{tabular}{|c|c|c|}
\hline MR Imaging Characteristic & Normal Peripheral Nerve & Abnormal Peripheral Nerve \\
\hline Size & $\begin{array}{l}\text { Decreases distally, similar or smaller than } \\
\text { accompanying artery }\end{array}$ & $\begin{array}{l}\text { Focal or diffuse enlargement, larger than } \\
\text { accompanying artery }\end{array}$ \\
\hline Signal intensity on T1WI & Isointense to skeletal muscle & Isointense to skeletal muscle \\
\hline $\begin{array}{l}\text { Signal intensity on fluid-sensitive sequences } \\
\text { (SPAIR, T2WIFS or STIR images) }\end{array}$ & $\begin{array}{l}\text { Isointense (SPAIR/fat sat T2WI) to minimal } \\
\text { hyperintensity (STIR) }\end{array}$ & $\begin{array}{l}\text { Moderate-to-marked hyperintensity approaching } \\
\text { fluid signal similar to that in adjoining veins }\end{array}$ \\
\hline Fascicular pattern & $\begin{array}{l}\text { Seen on both T1WI and T2WI, especially in larger } \\
\text { nerves }\end{array}$ & $\begin{array}{l}\text { Enlarged single or multiple fascicles, loss of normal } \\
\text { fascicular pattern }\end{array}$ \\
\hline Course & $\begin{array}{l}\text { Well-outlined by perineural fat, which is best } \\
\text { seen on T1WI }\end{array}$ & Deviated from normal course \\
\hline Enhancement with intravenous gadolinium & No appreciable enhancement ${ }^{\mathrm{a}}$ & $\begin{array}{l}\text { Abnormal enhancement when blood-nerve barrier is } \\
\text { breached }\end{array}$ \\
\hline Indirect signs, muscle denervation changes & Absent & Muscle edema or fatty atrophy may be present \\
\hline
\end{tabular}

a Exception: enhancement may be seen in dorsal nerve root ganglia of normal peripheral nerves, where blood-nerve barrier is absent.

\section{High-Resolution MRN Technique}

MRN techniques can be broadly divided into T2-based imaging or diffusion-based imaging. T2-based MR imaging of peripheral nerves is generally preferred over diffusion-based imaging because radiologists and technologists are more familiar with T2-based imaging; moreover, the techniques and the pulse sequences are easier to implement. ${ }^{5}$ Diffusion-based imaging of peripheral nerves is still in the research phase due to suboptimal SNR obtained in peripheral nerve imaging and at present has not been widely implemented. ${ }^{6,7}$ T2-based MRN imaging relies on obtaining high-resolution (2- to $3-\mathrm{mm} \mathrm{sec}-$ tion thickness) T1WI SE/T1WI FLAIR images and T2WIFS SE/SPAIR/STIR images in multiple planes (Fig 1). The abundant fat around the fascicles and the nerve itself makes these structures clearly visible on T1WI. STIR works best at $1.5 \mathrm{~T}$, while SPAIR with varying flip angles is the favored fat-suppression technique for 3T magnets because it provides higher SNR than STIR images. ${ }^{1}$ T2WIFS may provide inhomogeneous fat suppression, especially for large peripheral areas of the body chosen for evaluation of diffuse nerve lesions. 3D pulse sequences such as SPACE have been reported to perform well at $1.5 \mathrm{~T}$ and $3 \mathrm{~T}$ MR imaging (Fig 2). ${ }^{8}$ Multiplanar reconstructions of these 3D sequences allow good depiction of peripheral nerves, which commonly course through a variety of obliquities.

\section{Normal and Abnormal Peripheral Nerves on High- Resolution MRN Imaging}

The reader should carefully evaluate various characteristics of peripheral nerves on high-resolution MRN imaging (Table 1), such as size, signal intensity, fascicular pattern (Fig 1), and course (Fig 2). Various imaging criteria are helpful in differ-

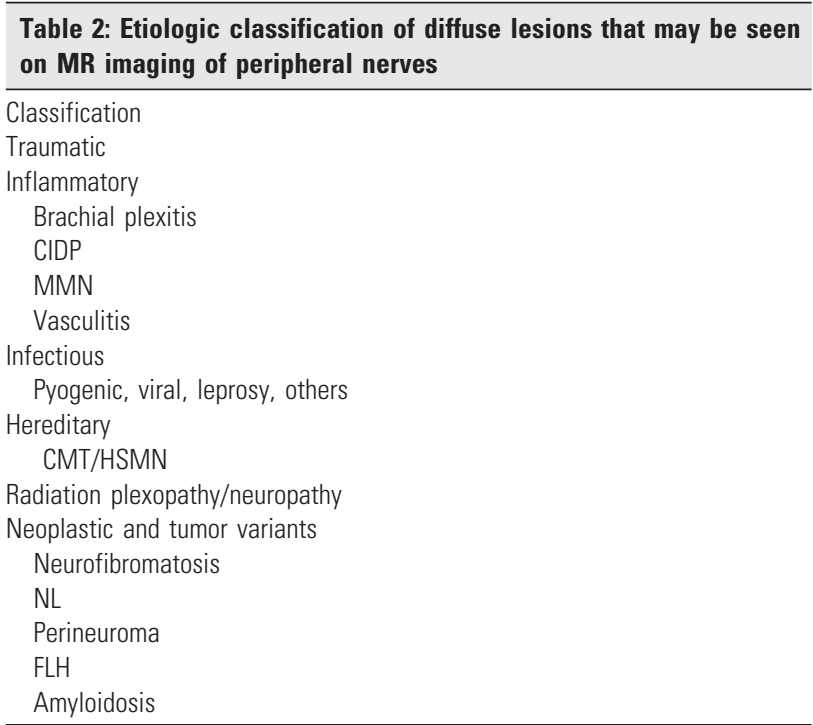




\begin{tabular}{|c|c|c|c|c|c|}
\hline Etiology & Enlargement & T2 Hyperintensity & $\begin{array}{l}\text { Fascicular } \\
\text { Pattern }\end{array}$ & Course & Enhancement \\
\hline Trauma & \pm & + & $\begin{array}{l}\text { Minimally effaced/ } \\
\text { disrupted }\end{array}$ & $\begin{array}{l}\text { May be altered due to hematoma/ } \\
\text { fracture }\end{array}$ & - \\
\hline Acute inflammation & \pm & ++ & Effaced/preserved & Not altered & + \\
\hline CIDP & ++ & +++ & Effaced/enlarged & Not altered & ++ \\
\hline Infectious & $+/++$ & ++ & Effaced/disrupted & $\begin{array}{l}\text { May be altered by } \\
\text { abscess/granulation tissue }\end{array}$ & ++ \\
\hline CMT/HSMN & ++ & ++ & $\begin{array}{l}\text { Preserved } \uparrow \text { fatty } \\
\text { infiltration } \\
\text { around atrophic } \\
\text { fascicles }\end{array}$ & Normal & \pm \\
\hline Radiation & $\begin{array}{l} \pm \text { (linear; geographic } \\
\text { distribution of } \\
\text { radiation field) }\end{array}$ & + & Preserved/effaced & $\begin{array}{l}\text { Altered in subacute-chronic stages } \\
\text { due to developing fibrosis }\end{array}$ & + \\
\hline $\mathrm{NL}$ & ++ & ++ & Effaced/disrupted & Altered with focal masses & ++ \\
\hline NF1 & ++ & ++ & Preserved & Altered with focal masses & $+/++$ \\
\hline MPNST in NF1 & +++ & + , Necrotic areas & Disrupted & Altered due to mass-effect & ++ (heterogeneous) \\
\hline Perineuroma & ++ & ++ & $\begin{array}{l}\text { Preserved/effaced } \\
\text { focally }\end{array}$ & Altered with focal masses & ++ \\
\hline FLH & +++ & $\begin{array}{c}+ \text { (fat causing } T 1 \\
\text { hyperintensity) }\end{array}$ & $\begin{array}{l}\text { Preserved (coaxial } \\
\text { cable/spaghetti) }\end{array}$ & Altered with thick tortuous nerve & - \\
\hline Amyloid & ++ & $\begin{array}{l}+ \text { Cutaneous induration with } \\
\text { subcutaneus stranding }\end{array}$ & $\begin{array}{l}\text { Preserved/disrupted } \\
\text { focally }\end{array}$ & Altered with focal masses & \pm \\
\hline
\end{tabular}

${ }^{\mathrm{a}} \pm$ indicates variable; + , mild; ++ , moderate; +++ , marked; - . absent.

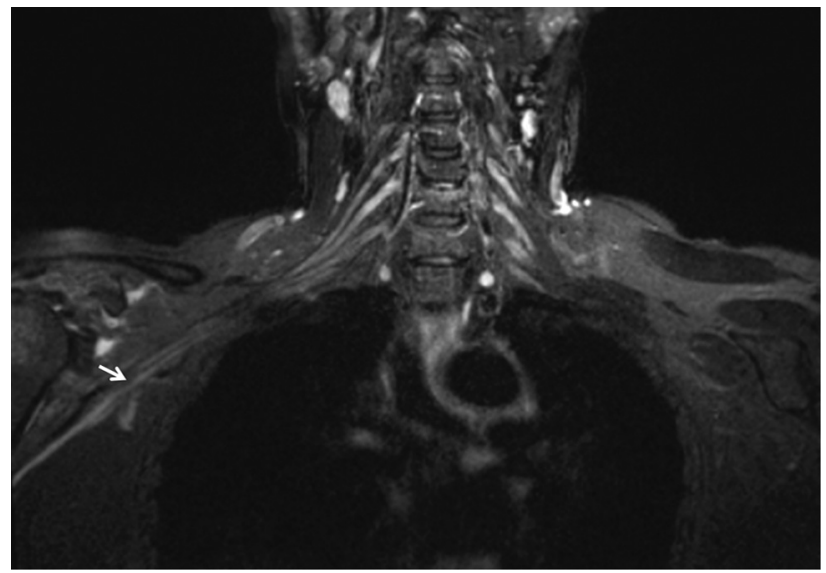

Fig 3. Traumatic neuropathy. A 46-year-old man had a posterior shoulder dislocation during a seizure episode. After the postictal phase, he had weakness of shoulder abduction and sensory loss over the deltoid area. Coronal STIR image shows minimal diffuse nerve enlargement and T2 hyperintensity of the terminal branch of the brachial plexus (arrow) as a result of direct trauma of the recent shoulder dislocation.

entiating a normal peripheral nerve from a diseased nerve (Fig 1). ${ }^{1,9}$ Intravenous administration of gadolinium is also helpful in detecting abnormal nerve and denervated muscle enhancement in patients suspected of various inflammatory, infectious, and neoplastic neuropathies.

\section{Etiology of Diffuse Peripheral Nerve Lesions}

Diffuse peripheral nerve lesions may be divided into traumatic, inflammatory, infectious, hereditary, radiation-induced, neoplastic, and tumor variants (Table 2). ${ }^{10-18}$ Relevant examples of cases from each category with their MRN imaging characteristics are illustrated (Table 3).

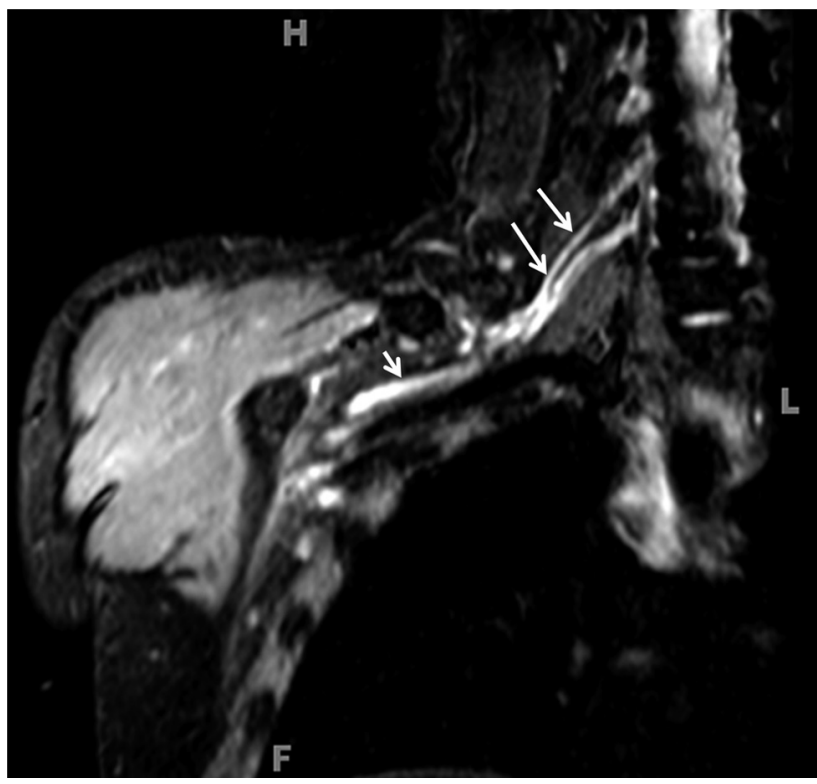

Fig 4. Brachial plexus neuritis. A 19-year-old man presented with acute onset of rightshoulder pain without any history of trauma. A week later the pain subsided, but weakness was noted on movement of the right shoulder. On examination, atrophy and severe weakness of abduction of the right shoulder (2/5, Medical Research Council scale for muscle power) were noted. Coronal T2WI shows T2 hyperintense and minimally enlarged right-sided C5 and C6 nerve roots (long arrows) and upper trunks (short arrow), in keeping with acute neuritis. Acute denervation changes were seen in the deltoid and suprascapularis muscles (image not shown).

\section{Traumatic Neuropathy}

Direct trauma to the peripheral nerve may be caused by stretching or laceration during a traumatic event. Other mechanisms of injury include acute or chronic compression related to hematoma, bony fracture, and ischemia and, rarely, intramuscular injections. ${ }^{10}$ Superficial nerves and fascicles that lie 

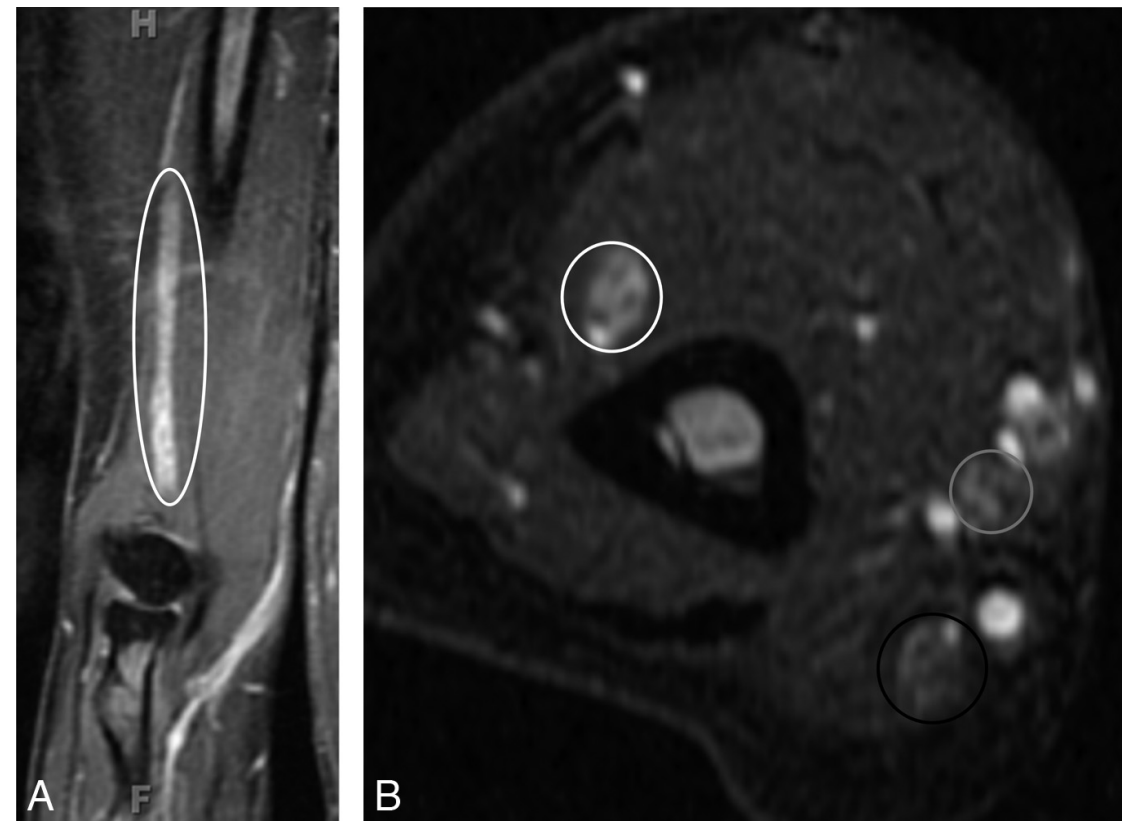

Fig 5. CIDP. A 55-year-old woman presented with a 1-year history of progressive pain in the hands and feet and weakness on finger extension of both hands. On examination, sensory loss and severe weakness in the bilateral distal radial nerve distribution were demonstrated. A nerve-conduction study found severe asymmetric sensory-motor peripheral neuropathy with demyelination (multifocal partial motor conduction block and reduced velocities). $A$, Coronal contrast-enhanced T1WIFS of the right upper extremity shows an enlarged and enhancing radial nerve (ellipse). The radial, median, and ulnar nerves were enlarged and T2 hyperintense bilaterally with contrast enhancement (images not shown). The diagnosis of CIDP was proved by biopsy in this case. B, Axial STIR image of the right upper extremity shows enlarged and hyperintense radial (white circle), median (gray circle), and ulnar (black circle) nerves. The changes are most pronounced in the radial nerve.
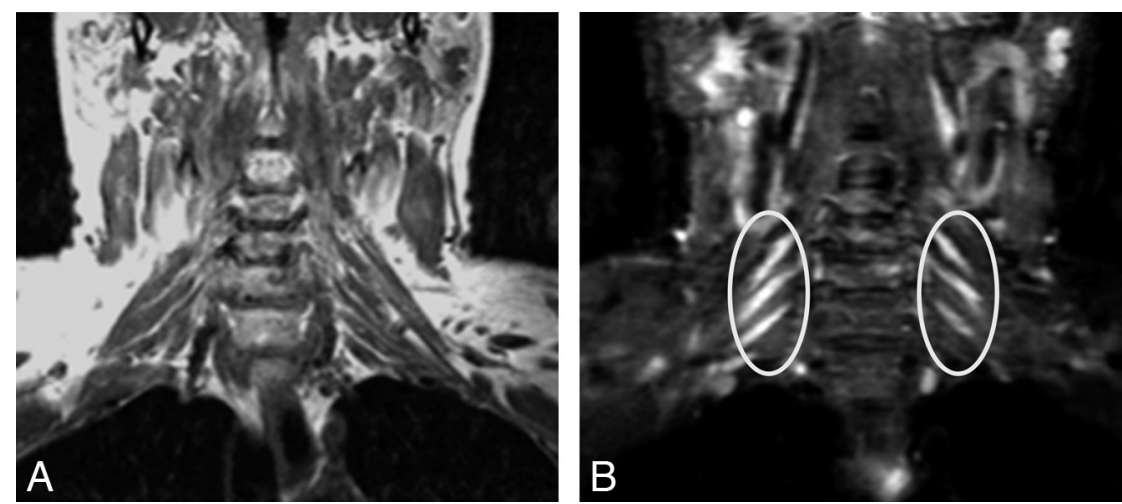

Fig 6. CIDP. A 46-year-old man had an acute episode of pain 2 years before imaging. The pain subsided, but he developed bilateral distal arm paresthesia. Clinical examination at the time of imaging showed distal sensory loss and motor weakness in both arms. Coronal T1 FLAIR (A) and coronal STIR (B) images show T2 hyperintense and symmetrically enlarged brachial plexus nerve roots and trunks (ellipses). The diagnosis of CIDP was proved by biopsy in this case.
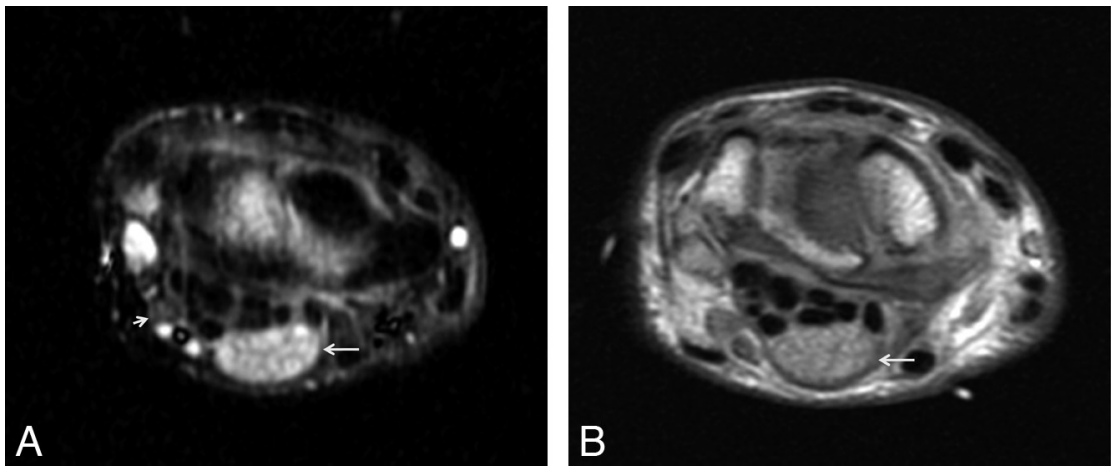

Fig 7. Infectious neuropathy (viral): Hansen disease (leprosy) in peripheral nerves. A 40-year-old man developed multiple small areas of lighter skin on the upper extremity 2 years before imaging. Subsequently, he developed weakness and decreased sensation on the arms and hands. T2WIFS $(A)$ and a proton-attenuation axial $(B)$ image show an enlarged hyperintense median nerve with prominent fascicles continuing into the carpal tunnel (arrows). The diagnosis of leprosy was proved by biopsy. 


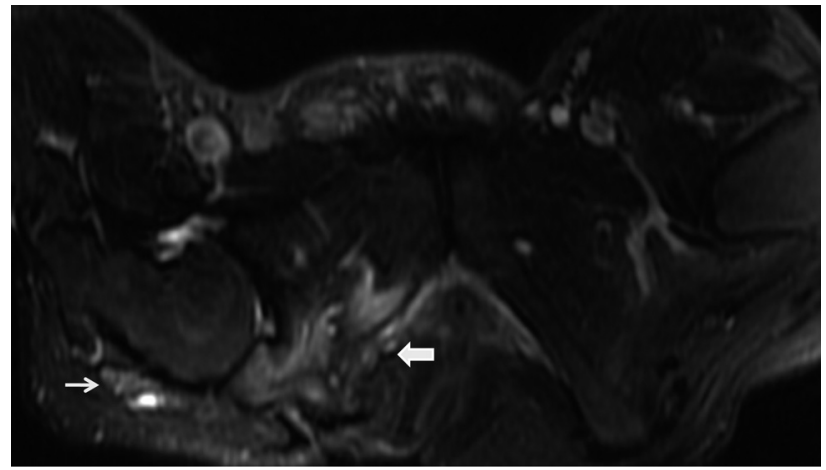

Fig 8. Infectious neuropathy (pyogenic). A 30-year-old man with a history of a long-standing right ischial decubitus ulcer and leg pain developed fever and chills with slow onset of sciatic neuropathy. MR imaging was performed for clinical suspicion of osteomyelitis. Axial T2WIFS shows an abnormally enlarged T2 hyperintense right sciatic nerve (thin arrow). Note the right ischial decubitus ulcer and osteomyelitis (thick arrow). The sciatic nerve demonstrated enhancement on postcontrast images (not shown). along the periphery of the nerve may be affected more severely in direct trauma, such as greater involvement of the peroneal component of the sciatic nerve by external trauma or compression. ${ }^{19}$ MRN may not only show the abnormal nerve (Fig 3 ), thereby confirming the clinical suspicion, but may also demonstrate the underlying causative lesion.

\section{Inflammatory Neuritis}

Peripheral nerves may be affected by a variety of inflammatory processes, and the clinically suspected pathologic nerves can be visualized in detail with high-resolution MRN imaging. Brachial plexus neuritis (plexitis) is a common occurrence. It is possibly immune-mediated and inflammatory, as is the form furste of acute GBS. ${ }^{11}$ The usual presentation is sudden onset of pain, followed by weakness and paraesthesia. ${ }^{20}$ There have been possible associations with viral or bacterial infection, vaccination, trauma, or surgery. This condition is self-
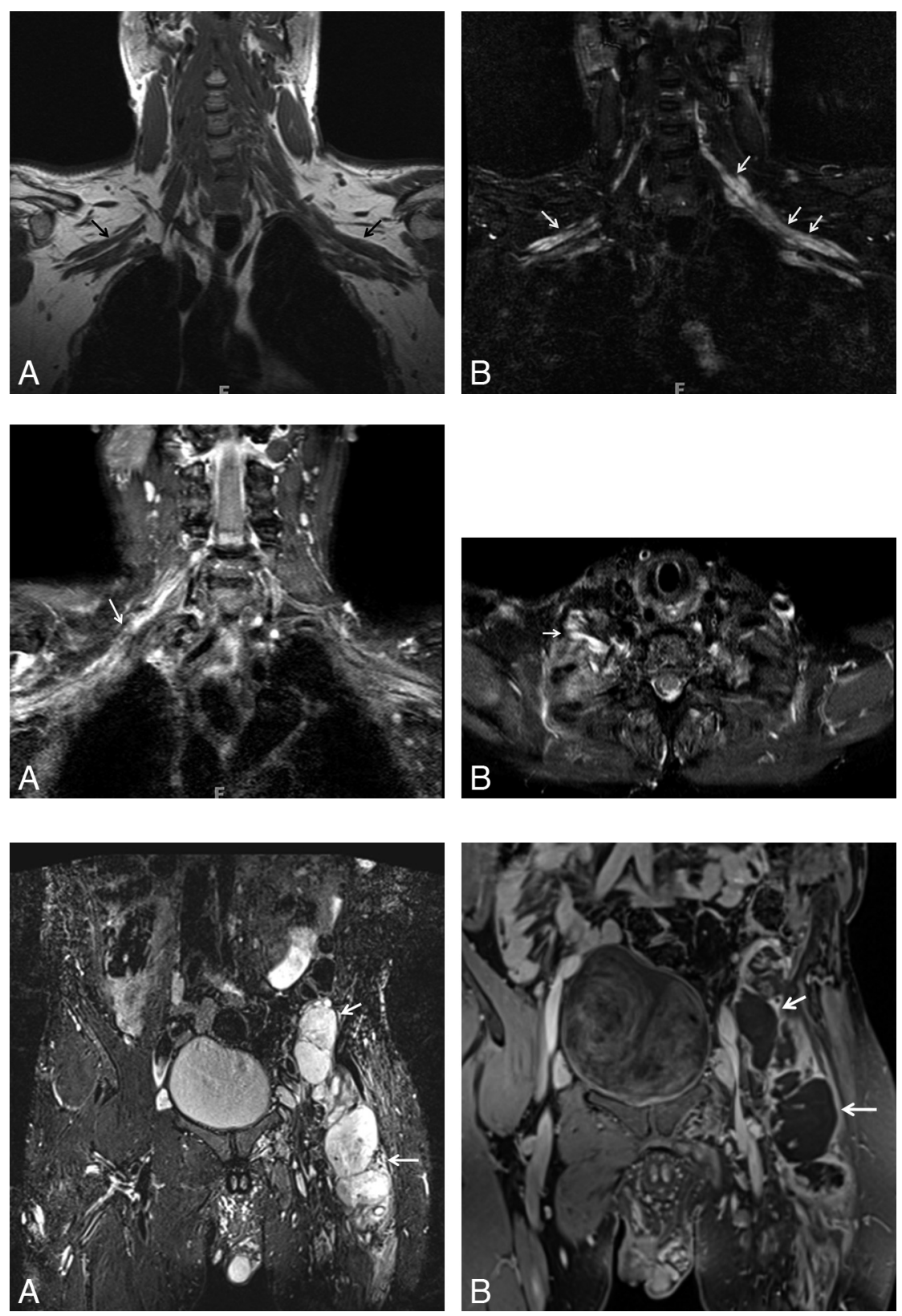

Fig 9. CMT. A 49-year-old man with progressive left hand and arm weakness and numbness, which started approximately 5 years before the current imaging. Coronal T1 FLAIR $(A)$ and coronal STIR $(B)$ images show hyperintense and enlarged brachial plexus nerve roots and trunks with a preserved fascicular pattern (arrows). There is asymmetric involvement of the brachial plexus in this case, with the left side more severely affected than the right side.

Fig 10. Radiation neuropathy. A 69-year-old woman with history of breast carcinoma and external beam radiation therapy 2 years before imaging presented with worsening right-arm sensory deficits. Coronal $(A)$ and axial $(B) 3 D$ STIR SPACE images show a T2 hyperintense right-sided brachial plexus (arrows) with minimal linear enlargement in the area of the radiation field. No focal mass is visualized.

Fig 11. Neurofibromatosis with MPNST. A 57-year-old man with neurofibromatosis presented with persistent left-leg pain and weakness. A, Coronal STIR image shows enlarged lobulated masses (arrows), which represent MPNST in this case. B, Coronal contrast-enhanced T1WIFS shows a heterogeneously enhancing lobulated mass with areas of necrosis along the left femoral nerve, demonstrating MPNST. 


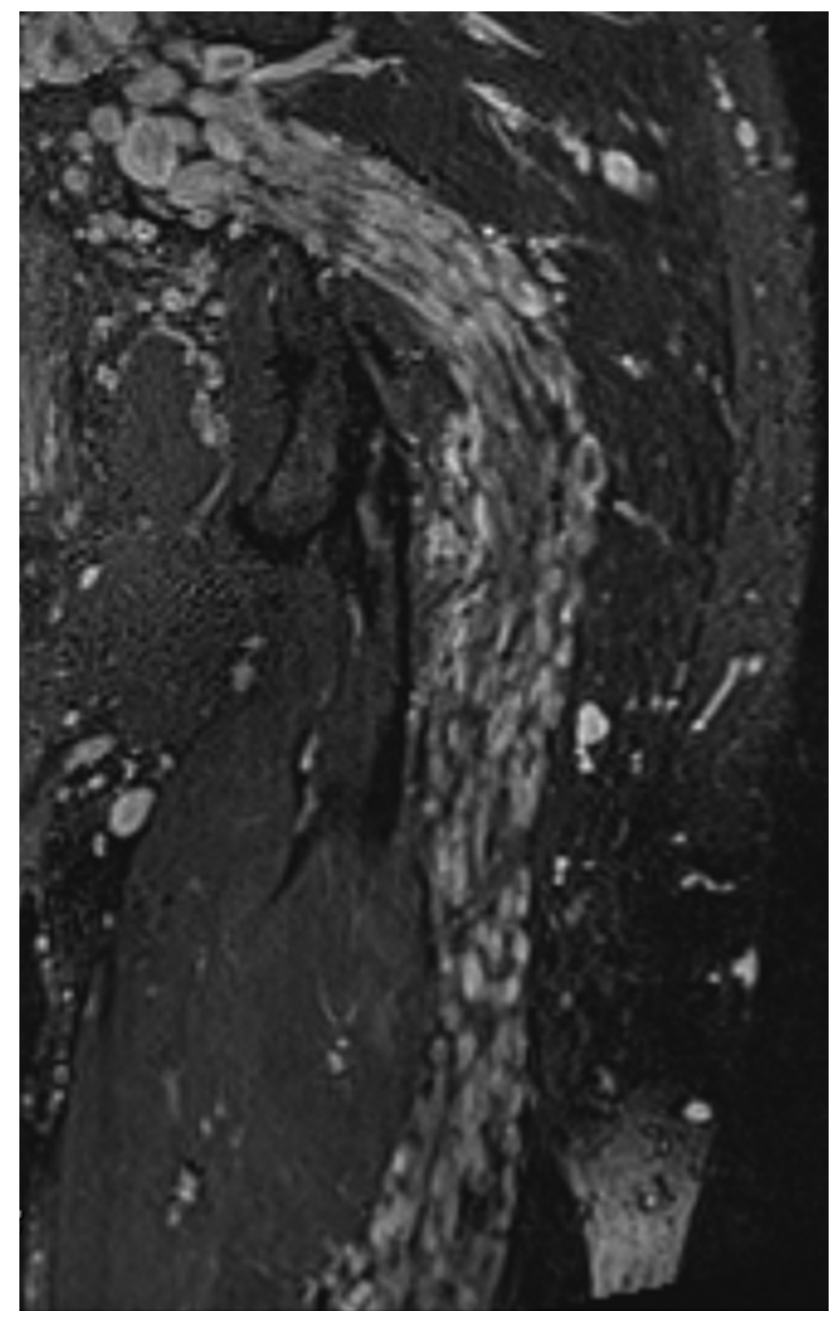

Fig 12. NF1. A 32-year-old man with history of neurofibromatosis underwent MR imaging for bilateral leg weakness. Coronal STIR SPACE image shows a diffusely enlarged left sciatic nerve with innumerable well-circumscribed small masses.

limiting (4-6 weeks), and treatment is conservative. MRN plays a crucial role in its management because it may confirm the diagnosis of brachial plexitis (Fig 4) as well as distinguish it from cervical radiculopathy. Surgery is needed for the latter diagnosis. Brachial neuritis involves multiple nerves, unlike the fixed nerve distribution of cervical spondylosis and degenerative disk disease.

\section{CIDP}

CIDP is rare and is considered the chronic counterpart of GBS; the peak of illness in GBS is $<4$ weeks. ${ }^{12,21}$ The clinical features include a chronic course ( $>2$ months); and proximal and distal muscle weakness with sensory involvement, areflexia, and albuminocytologic dissociation (Figs 5 and 6).

\section{Infectious Neuropathy}

Involvement of peripheral nerves in leprosy (Hansen disease, Fig 7) is well-known, and specific findings such as nerve calcifications in subacute-chronic phases may be seen. ${ }^{13,22}$ Pyogenic infections are rarer and may involve the nerve by direct extension (Fig 8).

\section{Hereditary Neuropathy}

CMT is a rare disease ${ }^{14}$; it is also known as hereditary motor and sensory neuropathy. A positive family history may be found in as many as $80 \%$ of cases. ${ }^{23}$ The usual clinical presentation is chronic degeneration of peripheral nerves and roots, with muscle atrophy and sensory impairment in a distal distribution. ${ }^{15,24}$ Clinical findings include atrophy and sensory loss affecting all extremities, ataxia, areflexia, palpably enlarged peripheral nerves, pes cavus, and hammer toes. Bilateral lumbosacral plexus and peripheral nerve involvement are common. The enlarged nerves are frequently related to increased fatty interfascicular epineurium with demyelinating atrophic fascicles (Fig 9).

\section{Radiation Neuropathy}

There may be a long and variable interval (weeks to years) between radiation therapy and the onset of symptoms. ${ }^{16}$ It is essentially a diagnosis of exclusion. Neoplastic disease (primary or metastatic), infectious, and other etiologies should be ruled out first (Fig 10). The severity is dose-dependent, and radiation neuropathy is seen more commonly with doses of $>60 \mathrm{~Gy}^{25}$
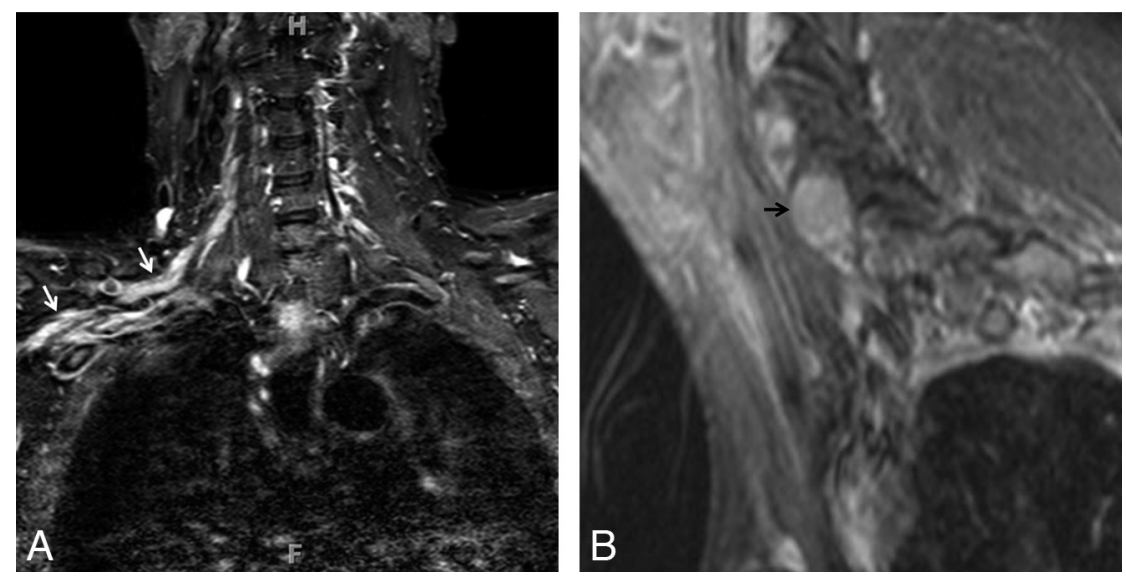

Fig 13. NL: Iymphomatous infiltration of the peripheral nerves. A 57-year-old man with B-cell Non-Hodgkin lymphoma presented with right C6 radiculopathy. A, Coronal STIR image shows multifocal segment enlargement of the right-sided brachial plexus (white arrows). B, Sagittal contrast-enhanced T1WIFS shows a focal enhancing mass at the C5-6 neural foramen (arrow). Images obtained after treatment did not show any residual disease (not shown). 

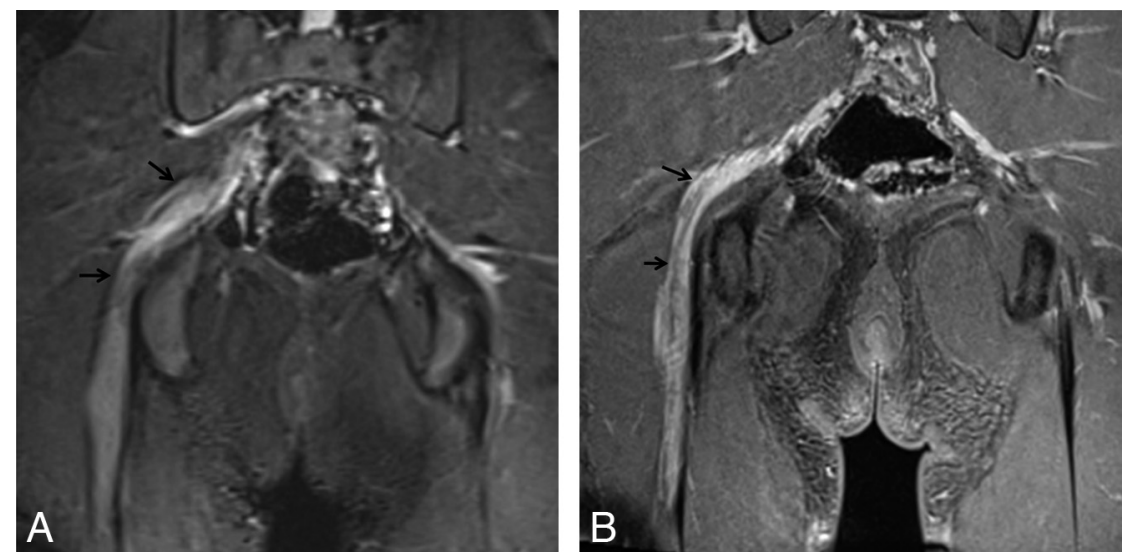

Fig 14. Perineuroma. A 16-year-old boy underwent MR imaging for right sciatic neuropathy with posterior thigh muscle atrophy and weakness. Coronal STIR image (A) and coronal contrast-enhanced $(B)$ T1WIFS show multifocal fusiform enlargement of the right sciatic nerve and enhancement (arrows). Perineuroma was proved by biopsy in this case.
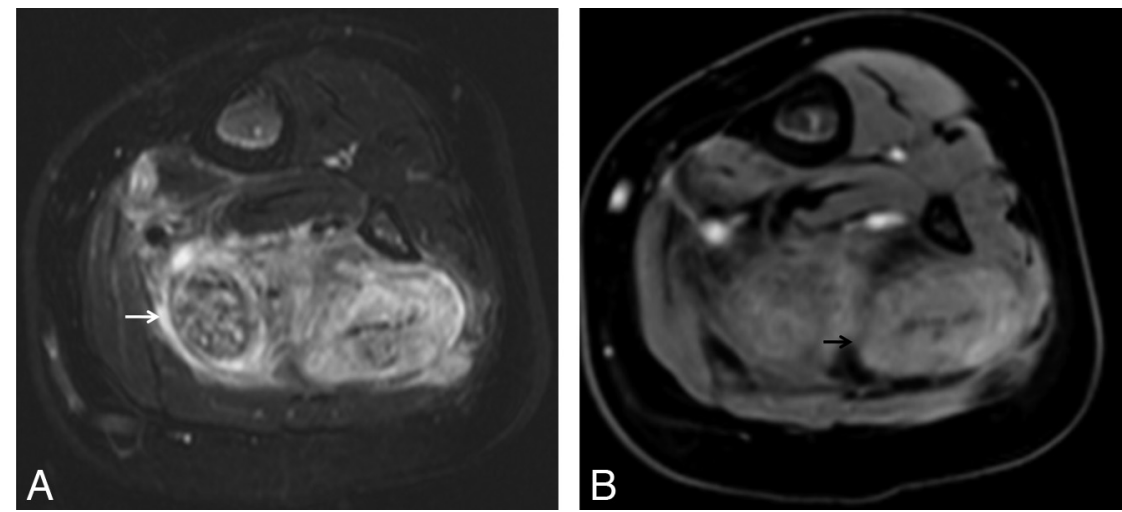

Fig 15. FLH. A 2-year-old boy presented with painless left calf and ankle swelling from 6 months before imaging. Examination of the left lower extremity revealed diffuse swelling throughout the medial aspect of the left calf and ankle. Axial T2WIFS $(A)$ and axial contrast-enhanced T1WI $(B)$ show a fat-containing nonenhancing mass of the tibial nerve with a coaxial cable appearance (arrows).
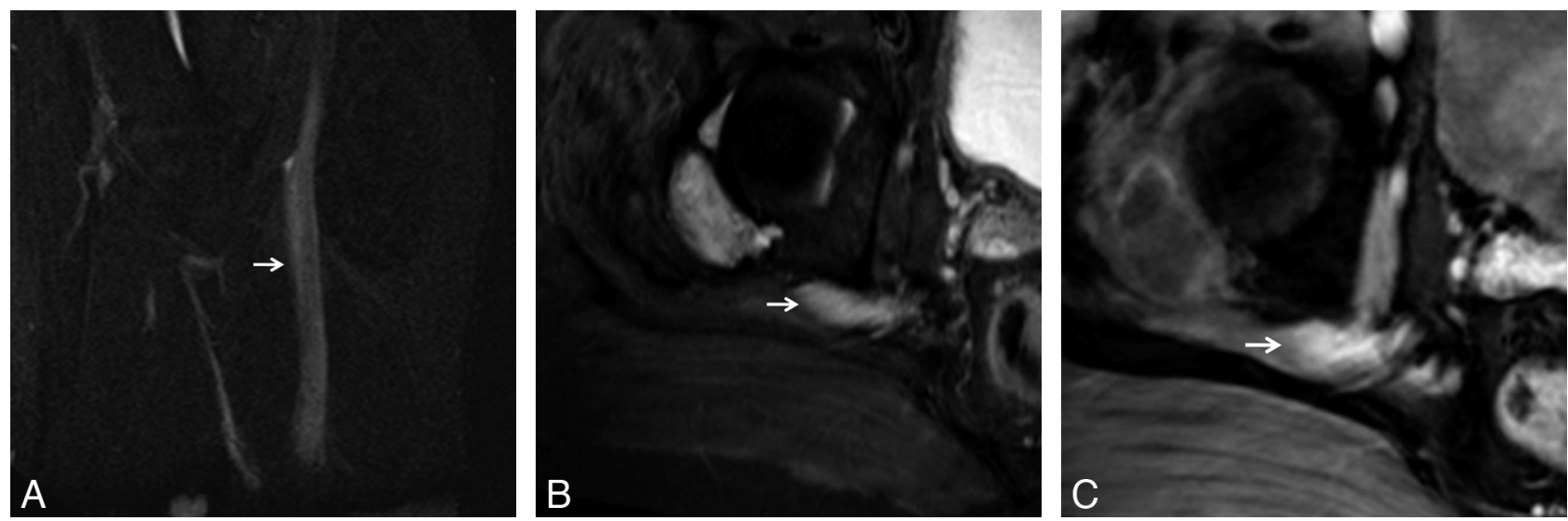

Fig 16. Amyloidosis of the peripheral nerve with amyloidoma. A 24-year-old man presented with a 1-year history of weakness in the right leg before imaging. There were no sensory symptoms. Electromyography and nerve-conduction studies revealed a deficit in the lumbosacral flexor distribution on the right side. $A$, Sagittal STIR image shows a diffusely enlarged T2 hyperintense right sciatic nerve (arrow). B, Axial STIR image shows localized swelling of the nerve consistent with amyloidoma (arrow). C, Axial contrast-enhanced T1WIFS shows diffuse enhancement in the abnormal sciatic nerve (arrow). Amyloidosis of the nerve was diagnosed by biopsy.

\section{Neoplasia and Tumor Variants}

A variety of tumors and tumor variants may involve the peripheral nerves. The role of MR imaging has been widely reported and is well-defined in neurofibromatosis (Figs 11 and 12). Multiple signs, such as a bag-of-worms appearance in plexiform neurofibroma, "split fat" sign on T1WI, and target and tail signs on T2WI, have been described. ${ }^{26,27}$ Other signs that may help to differentiate malignant from benign nerve sheath tumors are size $>5 \mathrm{~cm}$, necrosis or heterogeneous enhancement, and invasion of surrounding structures. ${ }^{26} \mathrm{NL}$ or lymphomatous infiltration of the peripheral nerves has been reported in $\leq 40 \%$ of patients dying of lymphoma. ${ }^{18}$ The typ- 
ical presentation is progressive sensorimotor neuropathy, and the diagnosis is usually confirmed by a histopathologic examination (nerve biopsy or autopsy) demonstrating lymphomatous infiltration of nerves (Fig 13).

Perineuroma is a benign tumor of neoplastic perineural cells (Fig 14). It may present as mononeuropathy or plexopathy. Motor symptoms with extensive chronic muscle denervation changes are more commonly seen than sensory symptoms. ${ }^{28,29} \mathrm{FLH}$ is a rare benign lesion, which has distinctive features on MR imaging. ${ }^{30,31}$ The median nerve is affected most commonly; however, it can also be seen at other sites (Fig 15). A variety of proteins may be deposited in nerves with systemic amyloidosis, resulting in severe progressive mixed neuropathy and autonomic dysfunction. ${ }^{17,32}$ The MR imaging appearance of amyloidosis of nerves has also been described (Fig 16). ${ }^{33}$

\section{Summary}

Diffuse abnormalities of peripheral nerves may be seen with numerous pathoetiologies. Currently, biopsy and pathologic examination of the nerves remain the only definitive ways of achieving a final diagnosis. However, a high-resolution MRN examination may be used to confirm the clinical suspicion and exclude other etiologies, such as a compressive mass lesion; in the correct clinical setting, it may aid in diagnosis and management of these lesions. Future research is needed to further validate the outlined differentiating MR imaging features of diffusely diseased peripheral nerves.

\section{References}

1. Chhabra A, Williams EH, Wang KC, et al. MR neurography of neuromas related to nerve injury and entrapment with surgical correlation. AJNR Am J Neuroradiol 2010;31:1363-68

2. Kim S, Choi JY, Huh YM, et al. Role of magnetic resonance imaging in entrapment and compressive neuropathy: what, where, and how to see the peripheral nerves on the musculoskeletal magnetic resonance image. Part 1. Overview and lower extremity. Eur Radiol 2007;17:139-49

3. Kim S, Choi JY, Huh YM, et al. Role of magnetic resonance imaging in entrapment and compressive neuropathy: what, where, and how to see the peripheral nerves on the musculoskeletal magnetic resonance image. Part 2. Upper extremity. Eur Radiol 2007;17:509-22

4. Amrami KK, Felmlee JP, Spinner RJ. MRI of peripheral nerves. Neurosurg Clin N Am 2008;19:559-72, vi

5. Kuntz C 4th, Blake L, Britz G, et al. Magnetic resonance neurography of peripheral nerve lesions in the lower extremity. Neurosurgery 1996;39:750-56, discussion 756-57

6. Zhang Z, Song L, Meng Q, et al. Morphological analysis in patients with sciatica: a magnetic resonance imaging study using three-dimensional highresolution diffusion-weighted magnetic resonance neurography techniques. Spine (Phila Pa 1976) 2009;34:E245-50

7. Yamashita T, Kwee TC, Takahara T. Whole-body magnetic resonance neurography. N Engl J Med 2009;361:538-39

8. Lichy MP, Wietek BM, Mugler JP 3rd, et al. Magnetic resonance imaging of the body trunk using a single-slab, 3-dimensional, T2-weighted turbo-spin-echo sequence with high sampling efficiency (SPACE) for high spatial resolution imaging: initial clinical experiences. Invest Radiol 2005;40:754-60

9. Hormann M, Traxler H, Ba-Ssalamah A, et al. Correlative high-resolution MRanatomic study of sciatic, ulnar, and proper palmar digital nerve. Magn Reson Imaging 2003;21:879-85

10. Plewnia C, Wallace $C$, Zochodne D. Traumatic sciatic neuropathy: a novel cause, local experience, and a review of the literature. J Trauma 1999;47:986-91

11. Sureka J, Cherian RA, Alexander M, et al. MRI of brachial plexopathies. Clin Radiol 2009;64:208-18

12. Koller H, Schroeter M, Kieseier BC, et al. Chronic inflammatory demyelinating polyneuropathy: update on pathogenesis, diagnostic criteria and therapy. Curr Opin Neurol 2005;18:273-78

13. Martinoli C, Derchi LE, Bertolotto M, et al. US and MR imaging of peripheral nerves in leprosy. Skeletal Radiol 2000;29:142-50

14. Berciano J, Combarros O. Hereditary neuropathies. Curr Opin Neurol 2003;16:613-22

15. Choi SK, Bowers RP, Buckthal PE. MR imaging in hypertrophic neuropathy: a case of hereditary motor and sensory neuropathy, type I (Charcot-MarieTooth). Clin Imaging 1990;14:204-07

16. Mondrup K, Olsen NK, Pfeiffer P, et al. Clinical and electrodiagnostic findings in breast cancer patients with radiation-induced brachial plexus neuropathy. Acta Neurol Scand 1990;81:153-58

17. Reilly MM, Staunton H. Peripheral nerve amyloidosis. Brain Pathol 1996;6:163-77

18. Krendel DA, Stahl RL, Chan WC. Lymphomatous polyneuropathy: biopsy of clinically involved nerve and successful treatment. Arch Neurol 1991;48:330-32

19. Yuen EC, So YT. Sciatic neuropathy. Neurol Clin 1999;17:617-31, viii

20. Miller JD, Pruitt S, McDonald TJ. Acute brachial plexus neuritis: an uncommon cause of shoulder pain. Am Fam Physician 2000;62:2067-72

21. Bradley LJ, Wilhelm T, King RH, et al. Brachial plexus hypertrophy in chronic inflammatory demyelinating polyradiculoneuropathy. Neuromuscul Disord 2006;16:126-31

22. Benedetti PF, Anderson MW, Maselli R, et al. Hypertrophic peripheral neuropathy due to leprosy: MR features. J Comput Assist Tomogr 1994;18:995-96

23. Emery AE. Population frequencies of inherited neuromuscular diseases: a world survey. Neuromuscul Disord 1991;1:19-29

24. Tachi N, Kozuka N, Ohya K, et al. MRI of peripheral nerves and pathology of sural nerves in hereditary motor and sensory neuropathy type III. Neuroradiology 1995;37:496-99

25. Petit-Lacour MC, Ducreux D, Adams D. MRI of the brachial plexus. J Neuroradiol 2004;31:198-206

26. Bhargava R, Parham DM, Lasater OE, et al. MR imaging differentiation of benign and malignant peripheral nerve sheath tumors: use of the target sign. Pediatr Radiol 1997;27:124-29

27. Li CS, Huang GS, Wu HD, et al. Differentiation of soft tissue benign and malignant peripheral nerve sheath tumors with magnetic resonance imaging. Clin Imaging 2008;32:121-27

28. Boyanton BL Jr, Jones JK, Shenaq SM, et al. Intraneural perineurioma: a systematic review with illustrative cases. Arch Pathol Lab Med 2007;131:1382-92

29. Merlini L, Viallon M, De Coulon G, et al. MRI neurography and diffusion tensor imaging of a sciatic perineuroma in a child. Pediatr Radiol 2008;38:1009-12

30. Nguyen V, Choi J, Davis KW. Imaging of wrist masses. Curr Probl Diagn Radiol 2004;33:147-60

31. Nilsson J, Sandberg K, Dahlin LB, et al. Fibrolipomatous hamartoma in the median nerve in the arm: an unusual location but with MR imaging characteristics-a case report. J Brachial Plex Peripher Nerve Inj 2010;5:1

32. Haridas A, Basu S, King A, et al. Primary isolated amyloidoma of the lumbar spine causing neurological compromise: case report and literature review. Neurosurgery 2005;57:E196, discussion E196

33. Metzler JP, Fleckenstein JL, White CL 3rd, et al. MRI evaluation of amyloid myopathy. Skeletal Radiol 1992;21:463-65 